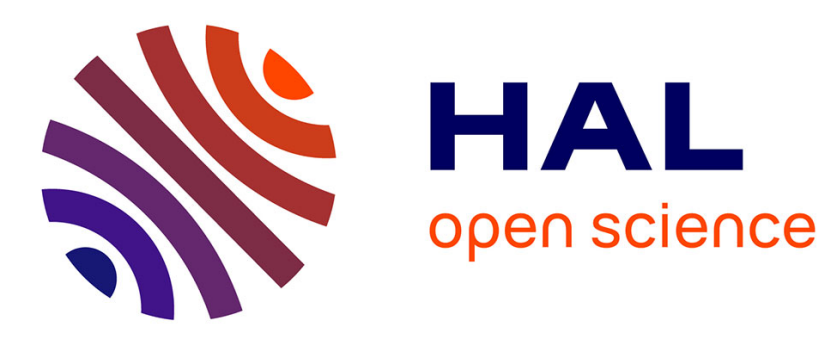

\title{
Locally induced laminar convection in liquid nitrogen and silicone oils
}

C Dubois, A Duchesne, B Vanderheyden, P Vanderbemden, H Caps

\section{To cite this version:}

C Dubois, A Duchesne, B Vanderheyden, P Vanderbemden, H Caps. Locally induced laminar convection in liquid nitrogen and silicone oils. European Physical Journal E: Soft matter and biological physics, 2016, 39 (8), pp.79. 10.1140/epje/i2016-16079-4 . hal-03329857

\section{HAL Id: hal-03329857 \\ https://hal.science/hal-03329857}

Submitted on 31 Aug 2021

HAL is a multi-disciplinary open access archive for the deposit and dissemination of scientific research documents, whether they are published or not. The documents may come from teaching and research institutions in France or abroad, or from public or private research centers.
L'archive ouverte pluridisciplinaire HAL, est destinée au dépôt et à la diffusion de documents scientifiques de niveau recherche, publiés ou non, émanant des établissements d'enseignement et de recherche français ou étrangers, des laboratoires publics ou privés. 


\title{
Locally induced laminar convection in liquid nitrogen and silicone oils
}

\author{
C. Dubois ${ }^{1}$, A. Duchesne ${ }^{1}$, B. Vanderheyden ${ }^{2}$, P. Vanderbemden ${ }^{2}$, H. Caps $^{1}$ \\ 1 GRASP, UR-CESAM - Physics Department B5, University of Liège, B-4000 Liège, Belgium. \\ 2 Montefiore, University of Liège, B-4000 Liège, Belgium.
}

June 27, 2016

\begin{abstract}
We present an experimental study of a laminar convective phenomena induced by a centimetric heater totally immersed in a liquid pool (Rayleigh number ranging from $2.10^{3}$ to $10^{7}$ ). This local heating is observed to induce a laminar convection that differs from the classical Rayleigh-Bénard cells created by heating the whole bottom of the fluid: the convection pattern is no more periodic. In order to obtain a complete map of the velocity field, we use Particle Image Velocimetry technique. The vertical velocity between the counter rotating convective cells is used as the relevant physical parameter to describe the phenomena. The potential cooling applications of this problem lead us to choose liquid nitrogen as an experimental fluid. We thus compare the results obtained for various temperature gradients in liquid nitrogen with experiments performed at room temperature with silicone oils of various viscosities. The theoretical law for the maximal vertical velocity from classical Rayleigh-Bénard experiments is adapted to the specific geometry investigated by using a new definition for the characteristic wavelength. This length is studied and appears to be dependent on the liquid properties. We finally obtain a remarkable agreement between theory and experimental data.
\end{abstract}

PACS. 4 4.25.+f, 47.20.Bp, 47.55.pb

\section{Introduction}

The convection created in a horizontal liquid layer heated from below was studied by Bénard [1] in 1901 and theoretically solved in the laminar case in 1913 by Rayleigh [2]. In particular, they observed and described periodic convective cells with a given wavelength, $\lambda$, fixed by the geometry. Numerous studies, both theoretical [3-7] and experimental $[8,9]$, improved and validated this theory.

Instead of an entire hot bottom, many practical and industrial applications imply local heaters. For instance, superconductor materials have to be immersed in a cryogenic liquid bath to be maintained in a superconductor state [10-12]. In regular use conditions, a superconductor puck may slightly heat, leading to laminar convection in the liquid pool. This situation where a local heater is immersed in a fluid is relevant in many cryogenic cooling processes [13-15].

Closer to these practical applications, numerous studies focused on the convection induced by a local heater attached on one of the liquid pool wall. Among others, the appearance of thermal plume [16-19] is reported, deformations of the free liquid surface [20] and pattern instabilities [21-23] are also observed. On the applied side, articles consider descriptions of velocity fields [15, 24, 25], transition to chaos [26,27], heating efficiency [28-30] but also study the effect of the heater length [24] or the multiplication of heaters [31] on the flow.

The case of a local heater far from any solid surface received much less attention, on both fundamental and applied aspects. In the present article, we focus on this case, trying to draw a link between this situations and the classical Rayleigh-Bénard case. Particularly, one can address the question of the convection vortices (number, positions, dependency upon the geometry or the fluid...) in this large aspect ratio object/surface configuration.

The fluid flow induced by the heater is optically investigated using a PIV technique within a home-made cryostat containing a large volume of liquid. Due to the potential cooling applications of the present work, liquid nitrogen is used. To compare the results obtained with this liquid, experiments with silicone oils of different viscosities are also performed. Liquid nitrogen was used at cryogenic temperatures, whereas silicone oils experiments were conducted at room temperature. The flow is characterized in terms of dimensionless number and the maximal vertical velocity above the heater is investigated as a function of the temperature difference between the heating source and the liquid pool. Previous theoretical predictions for this velocity are adapted to our specific geometry and a comparison between this model and the data is then proposed. 


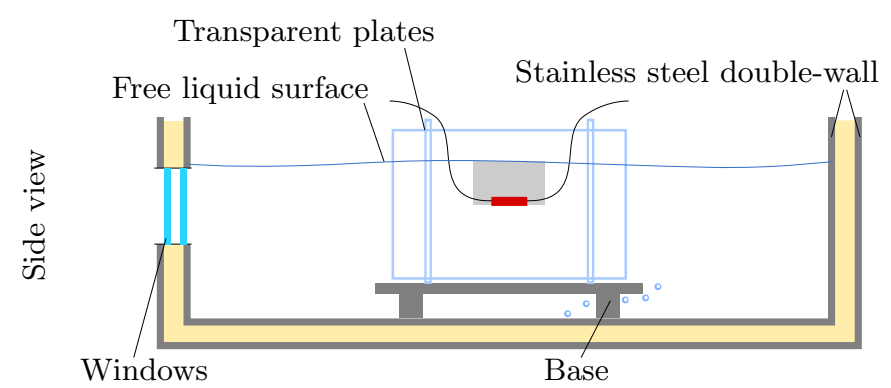

Figure 1. Experimental setup sketch. A heater (red rectangle) is immersed in an isolated volume of liquid. The grey rectangle corresponds to the typical field of view for PIV analysis.

\section{Experimental setup and procedure}

An electrical resistor $\left(10 \Omega, 6 \times 3 \times 1 \mathrm{~mm}^{3}\right)$ fed with an electrical current is used as heating source. The injected power responsible of the Joule's effect ranges in [0.1-2] W for all the experiments presented here. Copper wires are used to inject the current but also to maintain the resistor at a fixed location. We verified that the resistivity of those wires is so small that they do not significantly dissipate energy. The resistor is immersed in a liquid pool which constitutes an experimental fluid volume of $20 \times 3 \mathrm{~cm}^{2}$ as horizontal area and $10 \mathrm{~cm}$ in height. The resistor is located in the center of this volume, a few centimeters below the liquid free surface (see Fig. 1). Polycarbonate was chosen for building the box containing the liquid and the resistor.

The different liquids used in this study are liquid nitrogen and silicone oils $(0.65,10$ and $20 \mathrm{cS})$. The physical liquid characteristics, given by the provider (Dow Corning for the silicon oil and Air Liquide for liquid nitrogen), are listed in Table 1. As one can see, using these liquids allows us to vary the kinematic viscosity over a factor 30 , to double the surface tension but also the thermal diffusivity.

Thermalization of the liquid pool is ensured by immersing the polycarbonate box filled with the studied liquid into a large vessel which is thermalized. In practice, the experimental chamber is placed on small pillars in order to avoid perturbations from eventual bubbles nucleated on the cryostat bottom wall (see Fig 1). The whole set-up is placed on an optical table in order to avoid any vibrations. While the case of silicon oil is rather easy to realize, using liquid nitrogen led us to build a specific cryostat compatible with the PIV technique and allowing large liquid volumes $\left(15 \mathrm{dm}^{3}\right)$. For technical informations on this cryostat the reader may report to the Appendix. Before each experiment, the setup was left at rest a long time enough for the entire liquid to be thermalized.

For visualizing the liquid flow and performing PIV analysis, the experiment box has to be lighted through a side of the setup while images are taken through a perpendicular window. A commercial PIV (Dantec Dynamics) system is used to compute the velocity field of the flow on the basis of successive images of the liquid seeded with small tracers (a full description of this technique can be found in the literature, e.g. [32,33]). The technique is non-invasive even if it requires small tracer particles to be

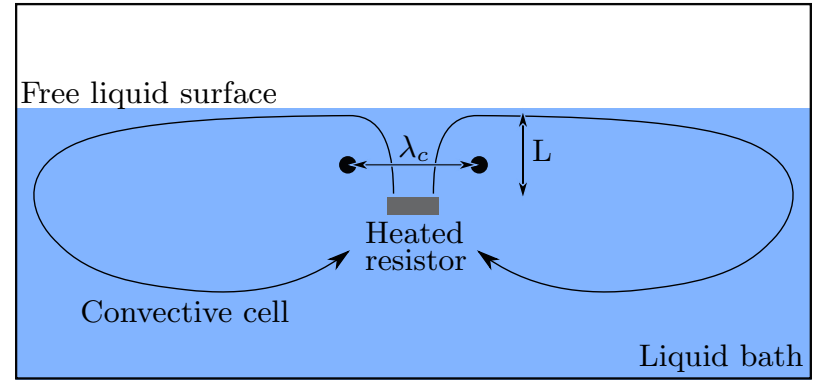

Figure 3. Sketch of the observed fluid motion. The heating resistor is immersed in the liquid bath at a distance $\mathrm{L}$ from the free surface. Two counter-rotating convective cells expand in the liquid bath. The rotation center of the cells are symbolized with dots, apart from a distance $\lambda_{c}$ defining the characteristic wavelength of the system.

seeded in the flow. These particles are chosen small enough to follow the flow without modifying it, but large enough to not undergo brownian motion. We used $\sim 15 \mu \mathrm{m}$ diameter polymer microspheres encapsulating a fluorescent dye (Rhodamine B) which showed a noticeable efficiency even at $77 \mathrm{~K}$ in liquid nitrogen. They were illuminated with a pulsed $532 \mathrm{~nm}$ laser. The laser beam is then shaped into a light sheet (thickness $\sim 0.1 \mathrm{~mm}$ ) in order to get two dimensional velocity fields. We verified that the energy injected by the laser in the system is so negligible that it does not induce any motion in the liquid. Images are acquired with a fast camera working at a frame rate of 100 fps. To obtain final 2D velocity fields, cross-correlation analysis are performed using a commercial software (DynamicSudio) on the experimental images.

A timelapse image obtained by superimposing many images used to performed PIV analysis is presented in Figure 2. The red rectangular area corresponds to the resistor. The top edge of the image is close to the free surface (1-2 $\mathrm{mm}$ ) while only part of the horizontal fluid volume is showed (see grey rectangle on Fig. 1).

\section{Flow overview}

On Fig. 2, one can distinguish two adjacent cells apart from a horizontal distance of the order of the heater width. The center of those cells, characterized by a null linear velocity, is closer to the free liquid surface than to the heater. A vertical motion of the liquid above the heater is also noticeable and is actually upward. Those observations are summarized in a sketch of the fluid flow in Figure 3.

In order to quantify those observations, we performed measurements of both the distance between the two cells and the maximal vertical velocity along a vertical line centered on the heater. The temperature difference, $\Delta \mathrm{T}$, between the heater and the liquid pool far from it is the main control parameter we considered. Two thermocouples (type $\mathrm{T}$ and PT 100) were used for measuring $\Delta \mathrm{T}$. The maximal temperature difference, $\Delta \mathrm{T}_{\max }=2 \mathrm{~K}$, for liquid nitrogen is mainly limited by its boiling point $(77.4 \mathrm{~K})$. Silicone oils are used at room temperature and the inter- 
Table 1. Kinematic viscosity $\nu$, density $\rho$, thermal expansion coefficient $\alpha$, thermal diffusivity $\kappa$ and surface tension $\gamma$ of the different fluids used in the present study.

\begin{tabular}{rrcrrr}
\hline Liquid & $\nu\left[\mathrm{m}^{2} \cdot \mathrm{s}^{-1}\right]$ & $\rho\left[\mathrm{kg} \cdot \mathrm{m}^{-3}\right]$ & $\alpha\left[\mathrm{K}^{-1}\right]$ & $\kappa\left[\mathrm{m}^{2} \cdot \mathrm{s}^{-1}\right]$ & $\gamma\left[\mathrm{N} \cdot \mathrm{m}^{-1}\right]$ \\
\hline Liquid nitrogen (77 K) & $210^{-7}$ & 810 & $510^{-3}$ & $810^{-8}$ & $910^{-3}$ \\
Silicon oil 0.65 cS (298 K) & $0.6510^{-6}$ & 905 & $10^{-3}$ & $410^{-8}$ & $2010^{-3}$ \\
Silicon oil 10 cS (298 K) & $1010^{-6}$ & 934 & $10^{-3}$ & $610^{-8}$ & $2010^{-3}$ \\
Silicon oil 20 cS (298 K) & $2010^{-6}$ & 950 & $10^{-3}$ & $910^{-8}$ & $2010^{-3}$ \\
\hline
\end{tabular}

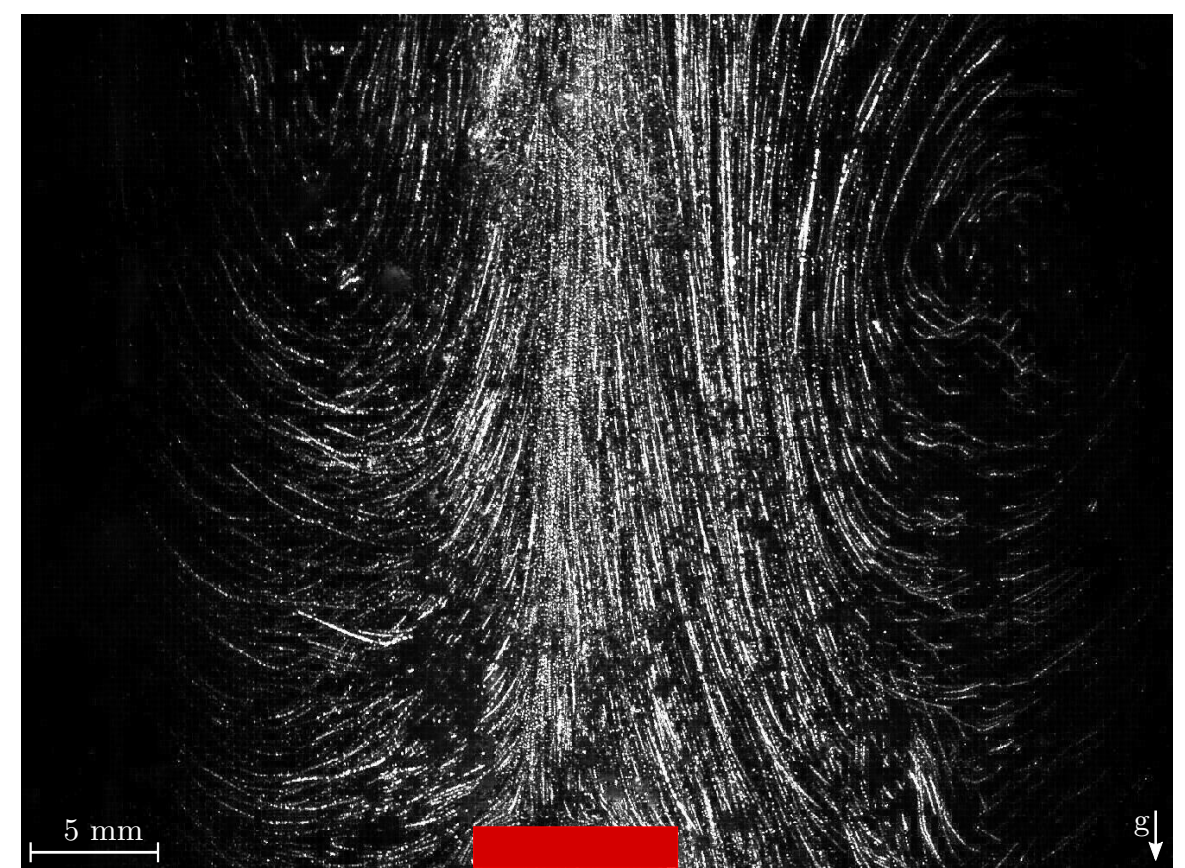

Figure 2. Timelapse image of the flow for $\Delta \mathrm{T}=2 \mathrm{~K}$ in liquid nitrogen. The horizontal resistor $\left(6 \mathrm{x} 3 \mathrm{x} 1 \mathrm{~mm}^{3}\right)$ is represented by the red rectangle area. An upward motion is induced above the heater. The top edge of the image is closed to the free surface (1-2 mm) and horizontally, only a portion is showed (see grey rectangle on Fig. 1).

val of temperature difference is of the order of a few tens of degrees. The $\Delta \mathrm{T}_{\max }$ for each liquid is given in Table 2 .

The fluid motion observed in Figure 2 results from a competition between the upward motion of lighter fluid elements generated by the heating source and viscous dissipation due to the relative motion of those rising elements and their surrounding fluid combined with the effect of thermal diffusivity spreading heat. The Rayleigh number is defined as the ration between those two effects and reads:

$$
\mathrm{Ra}=\frac{g \alpha \Delta \mathrm{TL}^{3}}{\kappa \nu}
$$

with $g$ the acceleration due to gravity, L the characteristic length of the system (see Fig. 3), $\rho, \kappa, \alpha$ and $\nu$ the density, the thermal diffusivity, the thermal expansion coefficient and the kinematic viscosity of the fluid respectively. These values are listed in Table 1.

As Ra exceeds a finite critical value $\mathrm{Ra}_{c}$, buoyancy overcomes dissipative effects. Theoretically, in the case of a horizontally layer heated from below, this happens for $\mathrm{Ra}_{c}=1707$ for perfect rigid walls [3-7]. In our experimental conditions, the heater geometry is different and the walls are not perfect. The latter shifts the instability appearance but has small effect as long as the horizontal to vertical ratio is large $[34,35]$, which is the case here $(\sim 10)$. Since that and the fact that our set-up did not allow us to accurately measure this threshold, we kept the cited value $\mathrm{Ra}_{\mathrm{c}}=1707$.

The tested Rayleigh numbers, listed in Table 2, are in $\mathrm{Ra} \in\left[10^{4}-10^{7}\right]$ for all the considered fluids. The lower values are larger than the critical $\mathrm{Ra}_{\mathrm{c}}$ while the larger ones are below the limit of convective turbulence (of the order of $10^{8}$, see for exemple $[36,37]$ ). In these conditions, the observed cells (see Fig. 2) can be characterized as convective ones. This range of $\mathrm{Ra}$ is quite wide and covers almost three decades. It corresponds to usual Rayleigh numbers investigated in discrete heaters experiments (e.g. [15, 24, 26, 27, 29-31]).

Two sources of convection are possible: (i) variations of the liquid density induced by a temperature difference (Rayleigh-Bénard instability) leading to a convective motion in the fluid and, (ii) variations of surface tension induced by a temperature difference (Marangoni-Bénard instability). The relative effect of Rayleigh to Marangoni 


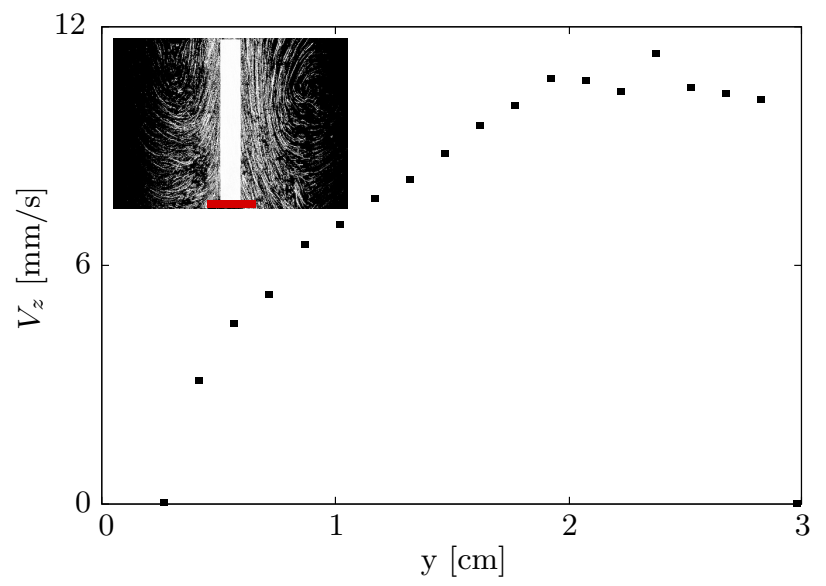

Figure 5. Vertical velocity, $V_{z}$, as a function of the vertical position in the central column (white rectangle in the inset) between the adjacent cells in liquid nitrogen.

effects can be compared using a Bond number defined as:

$$
\mathrm{Bo}=\frac{|\delta \rho| g \mathrm{~L}^{2}}{|\delta \gamma|},
$$

where $\delta \rho$ and $\delta \gamma$ stand for the variation of density with temperature and the variation of surface tension with temperature, respectively. This dimensionless number is close to 150 or larger for liquid nitrogen and roughly 270 and even larger for the three tested silicone oils (see Table 2). Under these conditions, Marangoni effect is negligible and Rayleigh-Bénard instability is considered as the motor of motion in the experiment.

\section{Vertical velocity}

As mentioned before, a pair of counter-rotating cells with a central upward column above the heating resistor appears in the fluid domain. These cells extend over all the available height between the resistor and the liquid free surface.

The core of the circulating rolls of each cell does not correspond to the center of half the experimental chamber on each side of the resistor, as visible in Figure 2 and sketched in Figure 3. The rotation centers (pointed by dots) are closer to the resistor than to the walls. Their locations correspond to the stagnation point in each cell, where the liquid has an absolute null velocity. In both numerical and experimental investigations found in the literature concerning laminar flows induced by local heaters located on walls, the same patterns are observed and the cell core positions are also observed to be uncentered (see $[15,24,26,27,29-31])$.

A typical velocity field in liquid nitrogen is presented in Figure 4 where the red rectangle at the bottom of the image corresponds to the heater and where the arrows give information on the fluid flow (direction and amplitude). It can be seen that the fluid vertical component of the velocity reaches its maximum in the central column between the two cells. According to the literature on the subject (see [9]), we focused on this maximal vertical velocity, $V_{z}$, between the two cells above the heating resistor and considered it as the relevant physical parameter describing the fluid flow. An example of velocity profile along the central column is presented in Figure 5. For the tested liquids and according to the experimented temperature differences, this maximal vertical velocity value, $V_{z}$, is reached a few millimeters bellow the free surface. With liquid nitrogen, the maximal velocity is reached some $5 \mathrm{~mm}$ bellow it.

The measured $V_{z}$ values are plotted in Figure 6 as a function of $\Delta \mathrm{T}$. The experimental points for liquid nitrogen and silicone oils appear in Figure 6(a) and (b) respectively. Each experiment was reproduced several times and vertical velocities were averaged to obtain statistical error bars as plotted in Figure 6 while errors on the temperature arise from the measurement accuracy. At fixed $\Delta \mathrm{T}$, velocities for liquid nitrogen are the largest.

A fit to the data reveals that all curves behave as a power law:

$$
V_{z} \sim \Delta \mathrm{T}^{\alpha}
$$

with $\alpha \simeq 0.5$ ( $\alpha=0.49$ for liquid nitrogen, 0.51 for $0.65 \mathrm{cS}$ oil, 0.53 for $10 \mathrm{cS}$ oil and 0.60 for $20 \mathrm{cS}$ oil).

\section{Interpretation}

The empiric power laws for $V_{z}(\Delta \mathrm{T})$ found in the literature rely on common Rayleigh-Bénard experiments with a bottom hot wall as heat source (e.g. [7-9]). Theoretical developments of local variable amplitudes in a convective fluid were given by Busse [6], while Norman et al. [7] performed a full calculation of the amplitudes and spatial dependencies of the velocity field for the Rayleigh-Bénard instability with Boussinesq approximation close to the onset of instability. Norman et al. [7] developed a theory for the maximal vertical velocity value close to the instability threshold. The distance from the instability onset is defined as $\frac{R a-R a_{c}}{R a_{c}}$ and serves as the relevant parameter for this modeling. The geometry considered by this model is a three-dimensional flow with rigid boundaries conditions and a horizontal dimension much larger than the height. Considering that the momentum diffusivity dominates leads to the following theoretical prediction:

$$
V_{z}=0.87 \sqrt{2}\left(\frac{2 \pi \mathrm{L}}{\lambda}\right)^{2} \frac{\kappa}{\mathrm{L}}\left(\frac{\mathrm{Ra}-\mathrm{Ra}_{\mathrm{c}}}{\mathrm{Ra}_{\mathrm{c}}}\right)^{1 / 2}
$$

where $\lambda$ is the wavelength of the periodic structure in the system.

Under these conditions, convection begins in form of rolls with alternating upward and downward flows. A regular cell pattern is formed with a clear wavelength $\lambda=2 \mathrm{~L}$ where $\mathrm{L}$ is the full cell height (see [5]). Here, as described above, the geometry leads to a different pattern with only two contra-rotative cells occupying the whole volume (see Fig. 4). We propose to adapt Eq(4) to this particular geometry by redefining the characteristic length $\mathrm{L}$ and the characteristic wavelength $\lambda$. 
Table 2. Temperature range $\Delta \mathrm{T}_{\max }$, Rayleigh number range Ra, Bond number Bo, characteristic length $\mathrm{L}$ and characteristic wavelength $\lambda_{c}$ for the reported experiments.

\begin{tabular}{rccccc}
\hline Liquid & $\Delta \mathrm{T}_{\max }[\mathrm{K}]$ & $\mathrm{Ra}$ & Bo & $\mathrm{L}[\mathrm{m}]$ & $\lambda_{c}[\mathrm{~m}]$ \\
\hline Liquid nitrogen & 2 & $210^{5}-7.010^{6}$ & $\geq 150$ & $3.010^{-2}$ & $(30 \pm 2) 10^{-3}$ \\
Silicon oil 0.65 cS & 10 & $110^{6}-1.410^{7}$ & $\geq 270$ & $2.510^{-2}$ & $(25 \pm 2) 10^{-3}$ \\
Silicon oil 10 cS & 30 & $810^{4}-2.510^{6}$ & $\geq 270$ & $2.510^{-2}$ & $(19 \pm 2) 10^{-3}$ \\
Silicon oil 20 cS & 35 & $410^{4}-1.410^{6}$ & $\geq 270$ & $2.510^{-2}$ & $(21 \pm 2) 10^{-3}$ \\
\hline
\end{tabular}

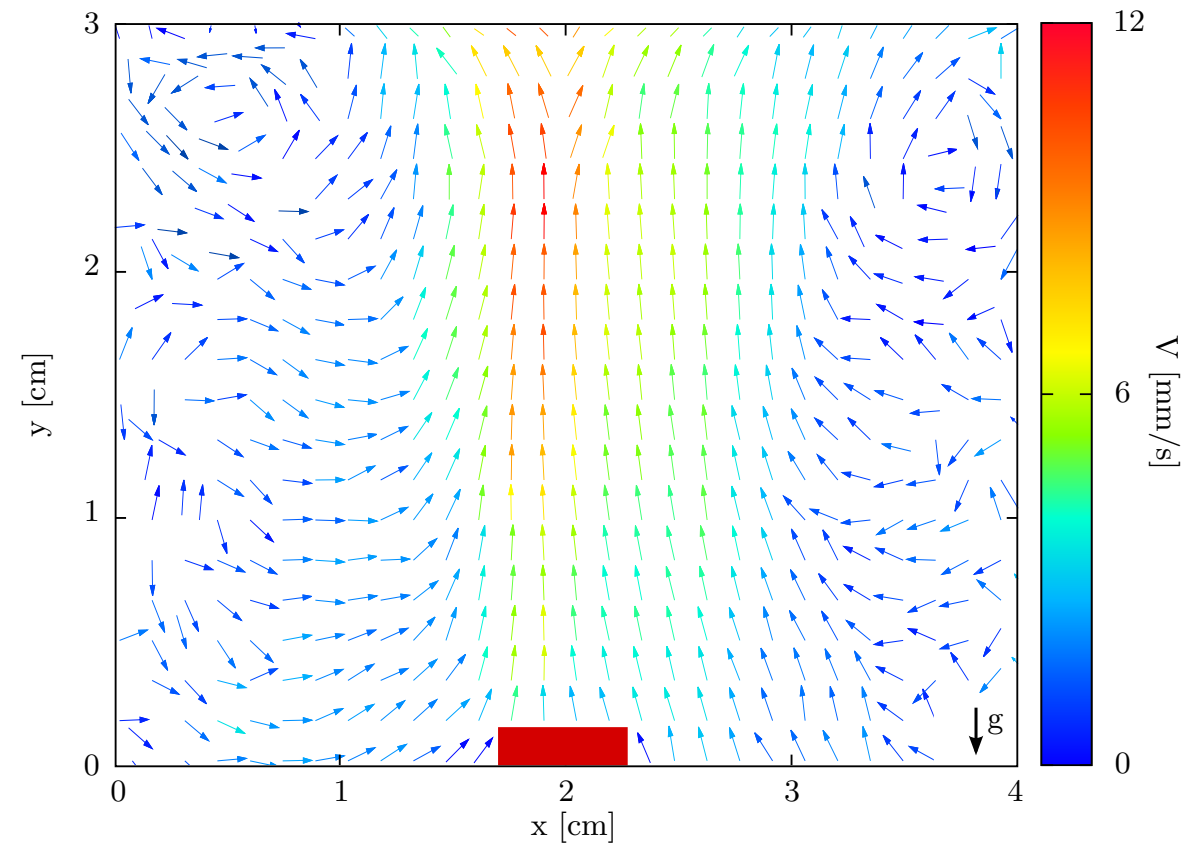

Figure 4. $2 \mathrm{D}$ velocity field above the heating source (red rectangle) for $\Delta \mathrm{T}=2 \mathrm{~K}$ in liquid nitrogen. The field of view is the same as in Figure 2.

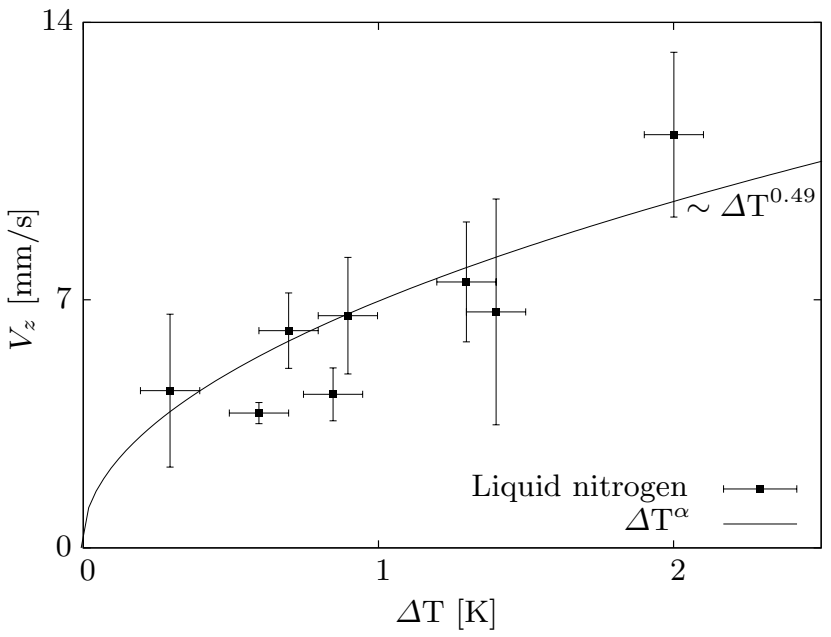

(a)

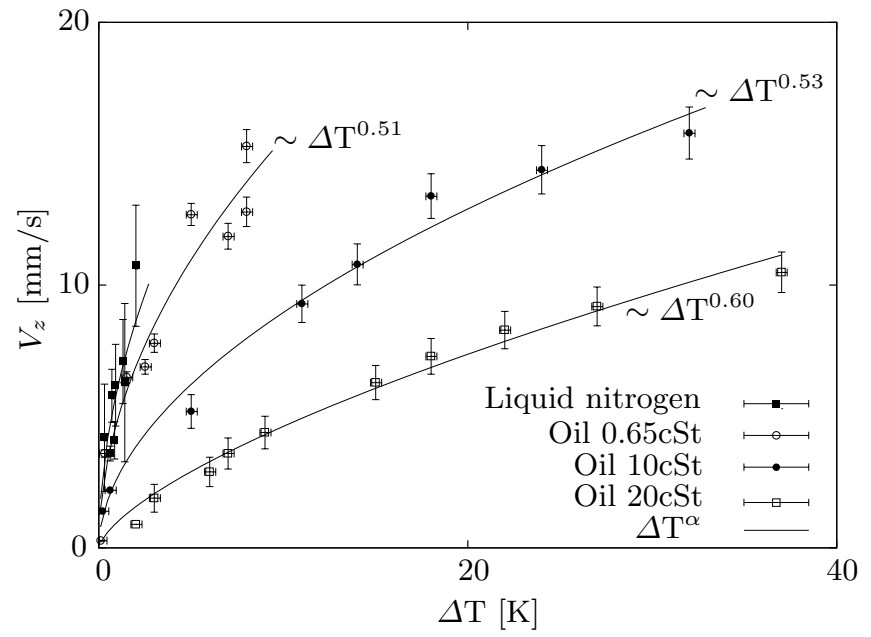

(b)

Figure 6. Vertical velocity, $V_{z}$, as a function of the temperature difference $\Delta \mathrm{T}$ in (a) liquid nitrogen alone and (b) liquid nitrogen and silicone oils. Solid lines correspond to fits to the data using $\mathrm{Eq}(3)$. 


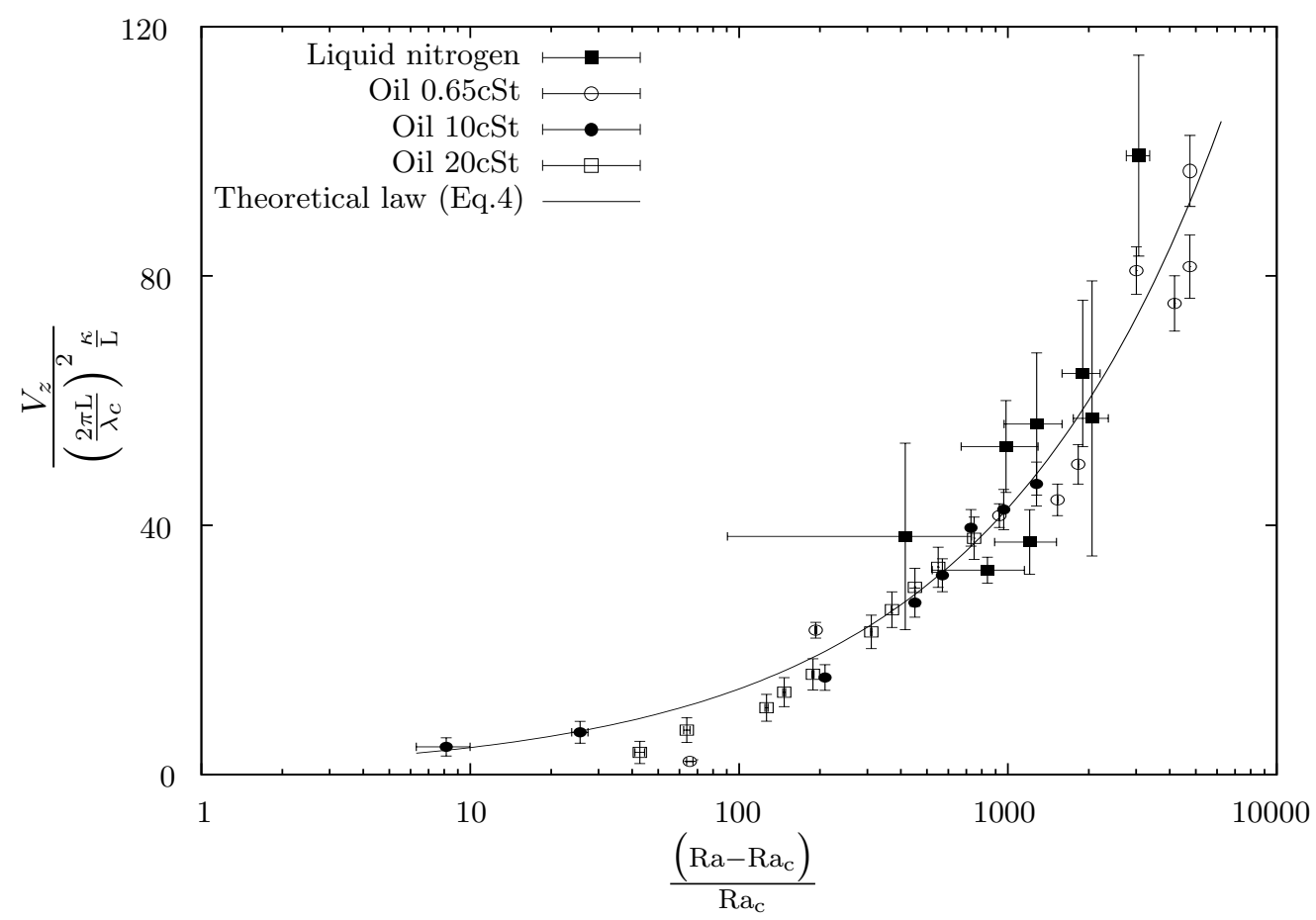

Figure 7. Dimensionless maximal vertical velocity as a function of $\frac{R a-R a_{c}}{R a_{c}}$ in semi-log scale. The solid line corresponds to $\mathrm{Eq}(4)$ without adjustable parameter.

First, the heater is immersed in the middle of the liquid pool, so the relevant characteristic length $\mathrm{L}$ can no more stand as the total liquid height. Since the liquid depth below the resistor is not observed to significantly influence the measured vertical velocity, the relevant length appears to be the distance between the heater and the liquid free surface. This approximation seems to be reasonable due to the nature of the instability responsible for the motion.

Second, we need to reconsider the characteristic wavelength $\lambda$ because we observe only two convective cells instead of a periodic pattern as in the classical RayleighBénard configuration. It is observed that the roll centers (corresponding to the stagnation points in each cell) do not match with the middle of the half experimental chamber, but are closer to the heating resistor. So, we suggest here to replace $\lambda$ by $\lambda_{c}$, the relevant wavelength of our system, defined as the horizontal distance between the two cell cores. This distance is observed to vary with the experiment geometry (for instance the length L and the cell thickness). A complete study of these variations is out of the scope of the present paper. The wavelength $\lambda_{c}$ also varies with the physical properties of the system such as the viscosity (see Table 2). The variations due to the temperature difference are such small that we cannot discriminate them and conclude on the relevance of the dependancy. These distances, extracted from PIV maps, are listed in Table 2.

Using the values for $\lambda_{c}$ and $\mathrm{L}$, experimental data are replotted with normalized axis in Figure 7. All data collapse on a single master curve and the model, without any fitting parameter, appears as a solid line. Given the measurement accuracy, the agreement between the proposed theory and the experimental data is remarkable for both kind of liquids. We find a behavior in $\left(\frac{R a-\mathrm{Ra}_{\mathrm{c}}}{\mathrm{Ra}_{\mathrm{c}}}\right)^{1 / 2}$ and all the physical parameters seem to be caught.

This comparison between data and theory suggests us two comments.

(i) The proposed definitions for $\lambda_{c}$ and $\mathrm{L}$ appear to be the relevant ones in order to describe the geometry investigated here. While it is not a surprise in the case of $\mathrm{L}$, even if this length does not take into account the extension of the convective cells below the height of the heater, the result for $\lambda_{c}$ is more surprising. It raises the question of the theoretical prediction of its value and the link between physical parameters and this wavelength which is different from the classical Rayleigh-Bénard experiment, where $\lambda$ is only predicted by geometry.

(ii) The presented model remains valid over a very wide range of Ra numbers. This is not a trivial result because $\mathrm{Eq}(4)$ is obtained with a perturbation development method and, in the limit of our experimental range, we are far away from the onset for the instability. To illustrate that fact, it is interesting to remind that Dubois et al. were surprised in [9] that the power law stands for $\frac{\mathrm{Ra}-\mathrm{Ra} \mathrm{a}_{\mathrm{c}}}{\mathrm{Ra}} \sim 10$. In our case, the result is still valid for $\frac{\mathrm{Ra}-\mathrm{Ra}_{c}}{\mathrm{Ra}_{c}} \sim 10^{4}$. The proposed model is thus relevant even close to the turbulent limit, which is $\mathrm{Ra} \sim 10^{7}-10^{8}[36,37]$. This might be explained by the fact that the flow is always observed as laminar. Moreover, the flow pattern composed of two convective cells is conserved over the whole range of $\mathrm{Ra}$ number we tested. 


\section{Conclusions}

We reported on the characteristics of the flow generated by a discrete heater immersed in a liquid pool. To observe the flow field in a cryogenic situation (use of liquid nitrogen as experimental fluid), we developed an optical cryostat able to be combined with PIV technique at low temperature. The analysis based on dimensionless numbers showed that the flow is laminar and convective. It also showed that the fluid motion is induced by the Rayleigh-Bénard instability. Two counter-rotating cells were observed in the experimental chamber. Moreover, the maximal vertical velocity between the two cells was studied as a function of the temperature difference between the heater and the liquid pool. Experiments were performed both in liquid nitrogen and in silicone oils of various viscosities. A theoretical expression for the maximal vertical velocity was derived from the literature and adapted to the specific geometry investigated here. In so doing, a new definition of the wavelength of the system, $\lambda_{c}$, and one of the relevant length, $\mathrm{L}$, were proposed. A very good agreement between experimental data and theoretical predictions was observed for both liquid nitrogen and silicone oils.

Quantitative observations of the global flow around the local heater presented in this study still contain a lot of information which should be investigated in the future; for instance the vorticity of the flow. The theoretical prediction of $\lambda_{c}$, which depends on the system geometry as in the classic Rayleigh-Bénard problem is still an open question. Beside the fundamental aspect, these questions present a great interest for cooling applications. Indeed, efficiency is linked to the flow rate of the convective flow, which is deeply correlated to $\lambda_{c}$ and to the vorticity. More generaly, the study of thermal convection close to and beyond the instability threshold is of fundamental interest.

\section{Acknowledgments}

This project has been financially supported by ARC SuperCool contract of the University of Liège. J.F. Fagnard is kindly acknowledged for fruitful discussions. M. Mélard and S. Rondia are particularly thanked for technical support.

\section{Appendix}

A double-wall optical cryostat (cfr. Figure 8) was designed using stainless steel as the main material for the container in order to ensure a good mechanical resistance to thermal shocks and to guarantee the absence of chemical interaction with the experimental liquid. Because stainless steel is a bad thermal insulator (thermal conductivity of $16 \mathrm{~W} \cdot \mathrm{m}^{-1} \cdot \mathrm{K}^{-1}$ ), we reinforced the insulation by injecting polystyrene in the gap between the walls. This foam has a low thermal conductivity $\left(0.03 \mathrm{~W} \cdot \mathrm{m}^{-1} \cdot \mathrm{K}^{-1}\right)$ and is able to fill all the space available. This foam being composed of air, gas bubbles could nucleate into the liquid if directly in

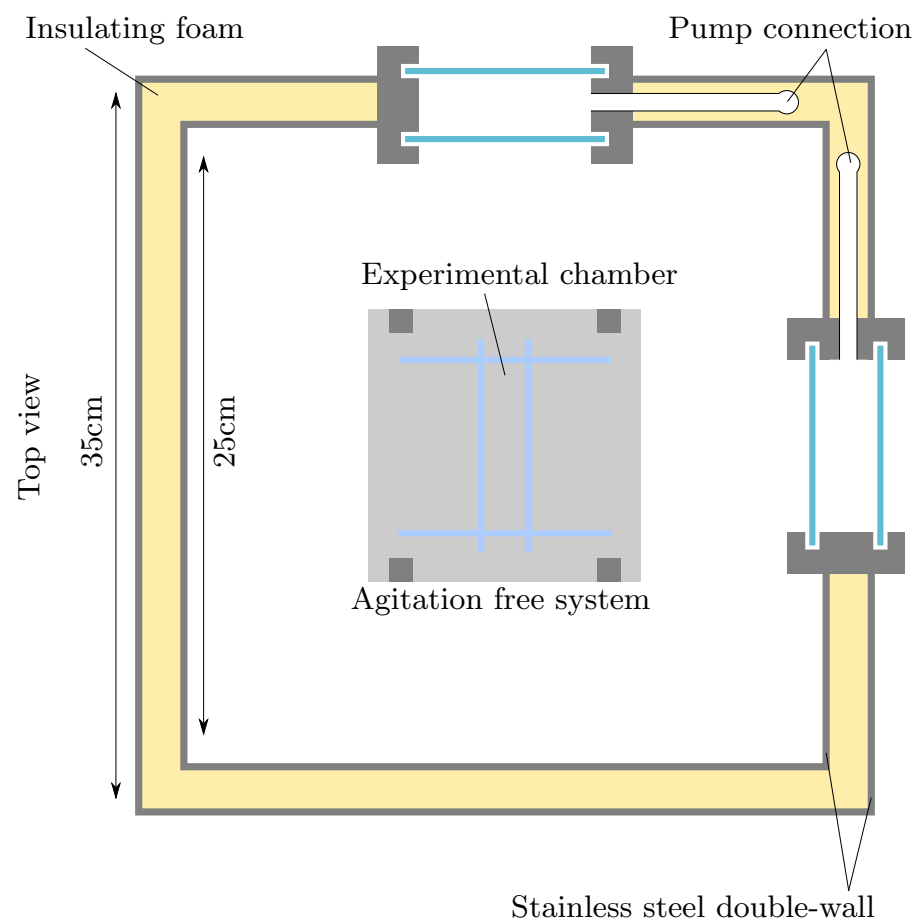

Figure 8. Sketch (top view) of the cryostat. Two stainless steel cubic boxes are wedged together. The first outer box has a side length of $35 \mathrm{~cm}$ while the second one his $25 \mathrm{~cm}$. Both boxes have $3 \mathrm{~mm}$ thick walls. The space between these boxes is thermally insulated with polystyrene foam. Two double-glazed windows (see Fig. 9 for more details) are imbedded in two adjacent sides of the cryostat. The inter-space between the two glasses is connected to a pump with a pipe imbedded in the insulant foam. An agitation-free system is placed at the center of the cryostat to reduce perturbations and large scale flows.

contact with it. The internal box prevents the appearance of this degassing risk.

The visualisation was made possible by fixing two doubleglazed windows (see Fig. 9) on two adjacent sides of the cryostat. Two $11 \mathrm{~cm}$ in diameter borosilicate disks were sandwiched between different $\mathrm{POM}^{\circledR}$ annular supports tough enough to seal the windows made of borosilicate glass. POM ${ }^{\complement}$ is a thermoplastic resistant at low temperature, resistant to thermal shocks and owning a small thermal expansion $\left(3.2510^{-6} \mathrm{~K}^{-1}\right)$. These properties are more important during the filling. The thermal expansion coefficient of the POM $\left(9.210^{-5} \mathrm{~K}^{-1}\right)$ being different from that of the borosilicate glasses, PTFE annular O-rings were placed between the two materials, allowing a good sealing of the cryostat. To avoid the apparition of condensation on the internal glass faces, a vacuum pump is connected to the inter-space between the double-glazing.

During the experiments, the top of the container is closed with a transparent polycarbonate plate in which holes are drilled in order to conserve the atmospheric pressure at the liquid/gas interface. Gas fills the space between the top of the liquid nitrogen free surface and the plastic panel roof of the cryostat. 


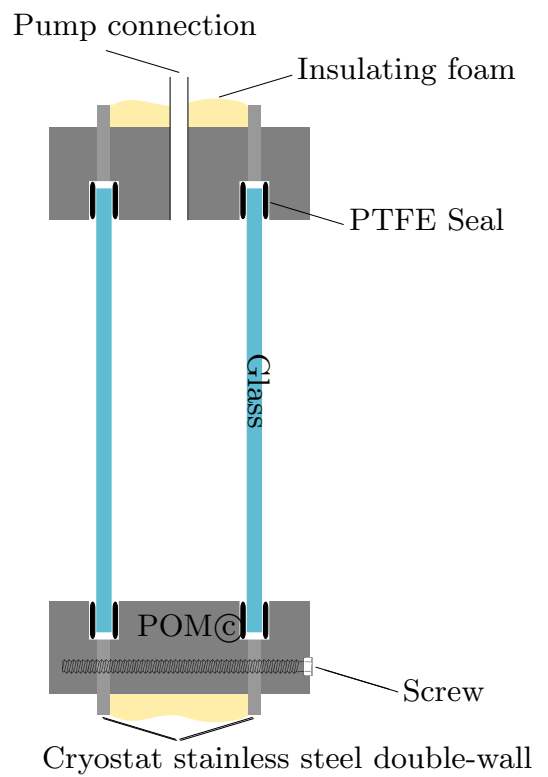

Figure 9. Double-glazed window (side view). Two circular glasses are sandwiched between POM rings. Four annular seals are placed between each POM annular and borosilicate glass to compensate their different thermal expansions. The gas present between the two glasses is removed by mean of a pump through a pipe imbedded in the insulant foam. The window is mechanically fixed with screws to the cryostat walls.

\section{References}

1. H. Bénard, "Les tourbillons cellulaires dans une nappe liquide. Méthodes optiques d'observation et d'enregistrement," Journal de Physique Théorique et Appliquée, vol. 10(1), pp. 254-266, 1901.

2. L. Rayleigh, "On convection currents in a horizontal layer of fluid, when the higher temperature is on the under side," The London, Edinburgh, and Dublin Philosophical Magazine and Journal of Science, vol. 32(192), pp. 529546,1916

3. A. Pellew and R. Southwell, "On maintained convective motion in a fluid heated from below," in Proceedings of the Royal Society of London A: Mathematical, Physical and Engineering Sciences, vol. 176, pp. 312-343, The Royal Society, 1940

4. W. Malkus and G. Veronis, "Finite amplitude cellular convection," Journal of Fluid Mechanics, vol. 4, no. 03, pp. 225-260, 1958.

5. A. Schlüter, D. Lortz, and F. Busse, "On the stability of steady finite amplitude convection," Journal of Fluid Mechanics, vol. 23, no. 01, pp. 129-144, 1965.

6. H. Busse, "On the stability of two-dimensional convection in a layer heated from below," Journal Math and Phys., vol. 46, pp. 140-150, 1967.

7. C. Normand, Y. Pomeau, and M. G. Velarde, "Convective instability: a physicist's approach," Reviews of Modern Physics, vol. 49(3), p. 581, 1977.

8. P. Berge and M. Dubois, "Convective velocity field in the Rayleigh-Bénard instability: experimental results," Physical Review Letters, vol. 32(19), p. 1041, 1974.

9. M. Dubois and P. Berge, "Experimental study of the velocity field in Rayleigh-Bénard convection," Journal of Fluid Mechanics, vol. 85(04), pp. 641-653, 1978.
10. V. S. Sharma, M. Dogra, and N. M. Suri, "Cooling techniques for improved productivity in turning," International Journal of Machine Tools and Manufacture, vol. 49(6), pp. 435-453, 2009.

11. K. D. Timmerhaus and T. M. Flynn, Cryogenic process engineering. Springer Science \& Business Media, 2013.

12. V. V. E. Schmidt, P. Muller, and A. V. Ustinov, The Physics of Superconductors: Introduction to Fundamentals and Applications. Springer Science \& Business Media, 1997.

13. B. Gebhart, Y. Jaluria, R. L. Mahajan, and B. Sammakia, Buoyancy-induced flows and transport. Springer, 1988.

14. S. Ostrach, "Natural convection in enclosures," Journal of Heat Transfer, vol. 110(4b), pp. 1175-1190, 1988.

15. S. M. Aminossadati and B. Ghasemi, "Natural convection cooling of a localised heat source at the bottom of a nanofluid-filled enclosure," European Journal of Mechanics-B/Fluids, vol. 28(5), pp. 630-640, 2009.

16. E. Moses, G. Zocchi, and A. Libchaberii, "An experimental study of laminar plumes," Journal of Fluid Mechanics, vol. 251, pp. 581-601, 1993.

17. D. Shlien, "Some laminar thermal and plume experiments," Physics of Fluids (1958-1988), vol. 19, no. 8, pp. 1089-1098, 1976.

18. M. C. Rogers and S. W. Morris, "Natural versus forced convection in laminar starting plumes," Physics of Fluids (1994-present), vol. 21, no. 8, p. 083601, 2009.

19. D. Funfschilling and G. Ahlers, "Plume motion and largescale circulation in a cylindrical rayleigh-bénard cell," Physical review letters, vol. 92, no. 19, p. 194502, 2004.

20. W. V. Kayser and J. C. Berg, "Surface relief accompanying natural convection in liquid pools heated from below," Journal of Fluid Mechanics, vol. 57, no. 04, pp. 739-752, 1973.

21. J. Vince and M. Dubois, "Hot wire below the free surface of a liquid: structural and dynamical properties of a secondary instability," EPL (Europhysics Letters), vol. 20, no. 6 , p. 505, 1992.

22. A. Mancho, H. Herrero, and J. Burguete, "Primary instabilities in convective cells due to nonuniform heating," Physical Review E, vol. 56, no. 3, p. 2916, 1997.

23. V. Erenburg, A. Y. Gelfgat, E. Kit, P. Bar-Yoseph, and A. Solan, "Multiple states, stability and bifurcations of natural convection in a rectangular cavity with partially heated vertical walls," Journal of Fluid Mechanics, vol. 492, pp. 63-89, 2003.

24. I. Sezai and A. A. Mohamad, "Natural convection from a discrete heat source on the bottom of a horizontal enclosure," International Journal of Heat and Mass Transfer, vol. $43(13)$, pp. $2257-2266,2000$.

25. I. L. Ngo and C. Byon, "Effects of heater location and heater size on the natural convection heat transfer in a square cavity using finite element method," Journal of Mechanical Science and Technology, vol. 29(7), pp. 29953003, 2015.

26. H. Koizumi, "Flow pattern formation and the transition to chaos in a confined container heated locally from below," International journal of thermal sciences, vol. 46(10), pp. 953-962, 2007.

27. X. Zhang, G. Su, J. Yu, Z. Yao, and F. He, "PIV measurement and simulation of turbulent thermal free convection over a small heat source in a large enclosed cavity," Building and Environment, vol. 90, pp. 105-113, 2015. 
28. I. E. Sarris, I. Lekakis, and N. S. Vlachos, "Natural convection in rectangular tanks heated locally from below," International journal of heat and mass Transfer, vol. 47(14), pp. 3549-3563, 2004.

29. N. B. Cheikh, B. B. Beya, and T. Lili, "Influence of thermal boundary conditions on natural convection in a square enclosure partially heated from below," International communications in heat and mass transfer, vol. 34(3), pp. 369-379, 2007.

30. C. Cianfrini, M. Corcione, E. Habib, and Q. A., "Convective transport in rectangular cavities partially heated at the bottom and cooled at one side," Journal of Thermal Science, vol. 22(1), pp. 55-63, 2013.

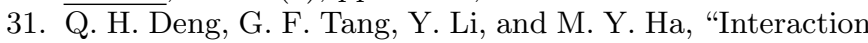
between discrete heat sources in horizontal natural convection enclosures," International Journal of Heat and Mass Transfer, vol. 45(26), pp. 5117-5132, 2002.

32. R. J. Adrian and J. Westerweel, Particle image velocimetry. Cambridge University Press, 2011.

33. M. Raffel, C. Willert, and J. Kompenhans, Particle Image Velocimetry: A Practical Guide. Springer, 1998.

34. I. Catton and D. K. Edwards, "Effect of side walls on natural convection between horizontal plates heated from below," Journal of Heat Transfer, vol. 89(4), pp. 295-299, 1967.

35. W. L. Heitz and J. W. Westwater, "Critical Rayleigh numbers for natural convection of water confined in square cells with L/D from 0.5 to 8," Journal of Heat Transfer, vol. 93(2), pp. 188-195, 1971

36. X. Chavanne, F. Chilla, B. Chabaud, B. Castaing, and B. Hebral, "Turbulent Rayleigh-Bénard convection in gaseous and liquid He," Physics of Fluids, vol. 13(5), pp. 1300-1320, 2001.

37. J. J. Niemela, L. Skrbek, K. R. Sreenivasan, and R. J. Donnelly, "Turbulent convection at very high Rayleigh numbers," Nature, vol. 404(6780), pp. 837-840, 2000. 